\title{
Methane hydrates achieve both thick oceans and thick lithospheres in Enceladus and Mimas
}

\author{
Ryusuke Nishitani ( $\nabla$ nishitani@ess.sci.osaka-u.ac.jp ) \\ Osaka University https://orcid.org/0000-0002-3763-0095 \\ Jun Kimura \\ Osaka University \\ Atsushi Tani \\ Kobe University \\ Sho Sasaki \\ Osaka University
}

Full paper

Keywords: Mimas, Enceladus, Subsurface ocean, Methane hydrate

Posted Date: January 28th, 2021

DOI: https://doi.org/10.21203/rs.3.rs-63051/v2

License: (c) (1) This work is licensed under a Creative Commons Attribution 4.0 International License.

Read Full License 


\section{Title page:}

2 Title: Methane hydrates achieve both thick oceans and thick lithospheres in

\section{$3 \quad$ Enceladus and Mimas}

4 Author \#1: *Ryusuke Nishitani, Department of Earth and Space Sciences, Graduate

5 School of Science, Osaka University, Toyonaka, Osaka, Japan, nishitani@ess.sci.osaka-

$6 \quad$ u.ac.jp

7 Author \#2: Jun Kimura, Department of Earth and Space Sciences, Graduate School of

8 Science, Osaka University, Toyonaka, Osaka, Japan, junkim@ess.sci.osaka-u.ac.jp

9 Author \#3: Atsushi Tani, Department of Human Environmental Science, Graduate

10 School of Human Development and Environment, Kobe University, Kobe, Hyogo,

11 Japan., tani@carp.kobe-u.ac.jp

12 Author \#4: Sho Sasaki, Department of Earth and Space Sciences, Graduate School of

13 Science, Osaka University, Toyonaka, Osaka, Japan, sasakisho@ess.sci.osaka-u.ac.jp

$14 \quad{ }^{*}$ corresponding author: nishitani@ess.sci.osaka-u.ac.jp 


\section{Abstract}

17 The difference between the inactive surface of Mimas and the active surface of

18 Enceladus is puzzling. We investigate the conditions under which both have a thick

19 subsurface ocean and the thermal lithosphere of Mimas is thicker than that of Enceladus

20 by using a one-dimensional simulation of thermal evolution. We adopt the initial core

21 temperature, initial methane concentration, and tidal heating rate as free parameters in

22 the calculation. The initial methane concentration and tidal heating rate greatly affect

23 the current ocean thickness, although the initial core temperature does not affect the

24 thickness. Methane hydrate forms at the base of the icy shell if the initial methane

25 concentration is not 0 . The methane hydrate layer plays an insulative role in an icy shell.

26 When the initial methane concentration is $1000 \mathrm{~mol} \mathrm{~m}^{-3}, \sim 2 \mathrm{GW}$ is needed to achieve

27 more than $50 \mathrm{~km}$ of the subsurface ocean on Mimas and $\sim 7.5 \mathrm{GW}$ is needed to achieve

28 more than $25 \mathrm{~km}$ of the subsurface ocean on Enceladus. These values are smaller than

29 those needed when the initial methane concentration is $0 \mathrm{~mol} \mathrm{~m}^{-3}$. The existence of the

30 methane hydrate layer promotes the survival of the subsurface ocean because it

31 insulates internal heat. In addition, it is found that the surface heat flux is depressed if 
32 the methane hydrate layer exists, which is consistent with the unrelaxed craters in

33 Mimas. Methane hydrate may explain the thick oceans in Mimas and Enceladus and the

34 inactive shell of Mimas.

\section{$35 \quad$ Keywords}

36 Mimas; Enceladus; Subsurface ocean; Methane hydrate

\section{$37 \quad$ Main Text}

\section{1. Introduction}

39 Cassini has revealed that Saturnian moons have various appearances, although their

40 surfaces are mainly covered with the same substance $-\mathrm{H}_{2} \mathrm{O}$ ice. This fact raises many

41 questions. One of the issues is the contrast between Enceladus and Mimas.

42 Enceladus has a high albedo surface. In the south polar region of Enceladus, an active

43 plume that includes ice particles and gaseous species is emitted (Porco et al. (2006)). A

44 composite infrared spectrometer (CIRS) aboard Cassini found high heat flow around the

45 region, and its power is estimated to be $\sim 15 \mathrm{GW}$ (Howett et al. (2011)). Gravity,

46 physical libration, and topography indicate that Enceladus has a global ocean beneath its

47 icy shell (Iess et al. 2014; Thomas et al. 2016; C̆adek et al. 2016). According to these 
data, the interior consists of a low-density core, thick ocean, and thin icy shell. The

49 plume is found to include nanosized silica particles and hydrogen, which indicates

50 ongoing hydrothermal activity at the seafloor (Waite et al., (2017); Hsu et al. (2015)).

51 These observations show that Enceladus is a hot and active body despite its small size.

52 In contrast to Enceladus, Mimas is dark. Many craters cover its surface. Although

53 absolute surface ages for Mimas and Enceladus are vague due to uncertainty of a

54 cratering rate, their crater densities indicate that Mimas surface is much older than most

55 of Enceladus surface (Kirchoff and Schenk (2009)). Additionally, there are few

56 fractures derived from geophysical activities. The largest crater, Herschell, is not overly

57 relaxed. Hence, Mimas is thought to be inactive. However, strong libration is observed,

58 which indicates a thick subsurface ocean or lumpy rocky core (Tajeddine et al. (2014)).

59 The phase lag of the libration supports the existence of a subsurface ocean. (Noyelles

60 (2017)). The suggestion of a subsurface ocean is confusing since craters on Mimas

61 should be more relaxed if the inside of Mimas is hot enough to have a global ocean

62 (Neveu and Rhoden (2017)). Rhoden et al. (2017) found that the tidal stresses evaluated

63 for the model of Mimas that included a deep ocean are hard to reconcile with Mimas' 
64 lack of tectonic activity. Hence, it is still unclear whether Mimas possesses a subsurface

65 ocean.

66 The physical and orbital properties of these two moons are summarized in Table 1.

67 Enceladus is larger in size and has a higher bulk density than Mimas. These differences

68 in properties are thought to produce differences in the thermal evolution of Mimas and

69 Enceladus and cause the contrast between Mimas and Enceladus. To constrain the

70 conditions to achieve the current internal structures and surfaces of Enceladus and

71 Mimas, it is essential to know the history of these moons. Therefore, we investigate the

72 long-term thermal evolution of each moon under various conditions.

73 The major heat sources needed to sustain global oceans are decay of long-lived

74 radioactive elements in rocky cores and tidal heating in the solid parts of the moons.

75 Although other heating sources may contribute, their heating rates are much lower than

76 the aforementioned major sources (Malamud and Prialnik 2013; Hand et al. 2011;

77 Nimmo et al. 2007). The total heat derived from radioactive decay depends on the rocky

78 core size, and those of Mimas and Enceladus are too small to melt ice shells and sustain

79 their subsurface oceans. Therefore, tidal heating is more important to maintain these 


$$
\dot{E}=-\frac{21}{2} \operatorname{Im}\left(k_{2}\right) \frac{\left(n R_{\mathrm{s}}\right)^{5}}{G} e^{2}
$$

83 where $\operatorname{Im}\left(k_{2}\right)$ is the imaginary part of the Love number, $G$ is the gravitational

84 constant, $n$ is the mean motion, $R_{\mathrm{s}}$ is the radius of the moon, and $e$ is the orbital

85 eccentricity (Segatz et al. (1988)). The heating rate is proportional to the square of

86 eccentricity. Since Mimas' orbital eccentricity is larger than Enceladus' orbital

87 eccentricity, depending on the value of $\operatorname{Im}\left(k_{2}\right)$, Mimas may have experienced enough

88 tidal heating to sustain the ocean. If $\operatorname{Im}\left(k_{2}\right)$ is small, it will not experience tidal

89 dissipation that would pull down the eccentricity. Generally, evaluating the Love

90 number is difficult because the value depends on how a moon deforms. On the other

91 hand, if two moons are in a mean motion resonance (MMR), the equilibrium tidal

92 heating rate can be calculated by considering the conservation of mass and angular

93 momentum (Meyer and Wisdom (2007)). The heating rate is described by

$$
\begin{gathered}
\dot{E}=\frac{n_{0} T_{0}}{\sqrt{1-e_{0}^{2}}}+\frac{n_{1} T_{1}}{\sqrt{1-e_{1}^{2}}}-\frac{T_{0}+T_{1}}{L_{0}+L_{1}}\left(\frac{G M m_{0}}{a_{0}}+\frac{G M m_{1}}{a_{1}}\right) \\
T=\frac{3}{2} \frac{G m^{2} R_{p}^{5} k_{2 p}}{a^{6} Q_{p}}
\end{gathered}
$$


96 where $T$ is the torque caused by the primary, $L$ is the angular momentum, $M$ is the

97 mass of the primary, $m$ is the mass of the moon, $Q$ is the tidal dissipation factor, and

$98 a$ is the semimajor axis. Subscripts 0 and 1 represent the inner moon and outer moon,

99 respectively. Subscript $\mathrm{p}$ represents the primary. Note that this equation does not rely on

100 the interior structure of the moon but on that of the primary (subscript $p$ ). The current

$101 Q_{\mathrm{p}}$ is suggested to be $\sim 2000$ from an astrometric study (Lainey et al. (2012)). At

102 present Enceladus and Dione are in a 2:1 eccentricity-type MMR. Assuming $Q_{\mathrm{p}}$ is now

1032000 , the equilibrium tidal heating rate for Enceladus is $\sim 10 \mathrm{GW}$. This tidal heating

104 rate is insufficient for the survival of a thick ocean (Kamata (2018)). Mimas is not

105 currently in any eccentricity resonance, but it is likely to have experienced eccentric

106 resonance in the past (e.g., Meyer and Wisdom (2008)). The most recent eccentric

107 resonance that Mimas may have experienced is 3:2 with Enceladus. Mimas may have

108 experienced this resonance $0.34 \mathrm{Gyr}$ ago, at which time $Q_{\mathrm{p}}$ was 4800 and the

109 equilibrium tidal heating rate was 3.6 GW (Tian and Nimmo, (2019)).

110 Dissolved ingredients in an ocean, such as ammonia, make it easier for the ocean to

111 survive because they depress the freezing point (Choukroun and Grasset (2010)). 
112 However, high concentrations of ammonia $(\sim 15 \mathrm{wt} \%)$ and tidal heating of $15 \mathrm{GW}$ are

113 needed to achieve an ocean of $\sim 25 \mathrm{~km}$ consistent with observations (Kamata (2018)),

114 and this is unlikely in Enceladus and Mimas since such amounts of ammonia were not

115 found in the Enceladus plume (Waite et al. (2017)).

116 Alternatively, we consider the existence of a methane hydrate layer beneath a pure ice

117 layer in an icy shell. Methane hydrate is a crystalline compound where methane gas is

118 included in a cage made of a hydrogen bond network of water (Sloan Jr and Koh

119 (2007)). Its appearance and some physical properties are similar to those of ice.

120 However, the thermal conductivity of methane hydrate is approximately four times

121 lower than that of ice at $263 \mathrm{~K}$ (Waite et al. (2009)), and its viscosity is one order of

122 magnitude higher than that of ice (Durham et al. (2003)). These two characteristics may

123 contribute to the thick subsurface ocean, as heat conduction and convection are

124 depressed by the low thermal conductivity and the high viscosity.

125 Additionally, this insulating layer lowers the temperature of the icy shell, so the icy

126 shell becomes more rigid than the shell which is composed of only ice. This rigid shell

127 has an advantage in the maintenance of the unrelaxed craters of Mimas. The assumption 
128 of a methane hydrate insulating layer succeeded in achieving both the maintenance

129 of the thick subsurface ocean and the ice shell thickness contrasts of Pluto

130 for a long time (Kamata et al. (2019)). In addition, Kalousová and Sotin (2020)

131 revealed that surface clathrate layer could prevent Titan's ocean freezing. We assume

132 that methane hydrate may similarly exist in Enceladus and Mimas. The plume

133 composition of Enceladus observed by Cassini includes not only water but also some

134 hydrophobic gases such as methane. With these plume compositions, gas hydrates can

135 form under the pressure and temperature of the subsurface ocean of Enceladus (Bouquet

136 et al. (2015)). If there are the methane hydrate layers in Mimas and Enceladus,

137 subsurface oceans of Mimas and Enceladus could survive with small amount of tidal

138 heating rate.

139 In this paper, we investigate the conditions under which both Enceladus and Mimas

140 have a thick inner ocean and where Mimas is less active than Enceladus, which means

141 that Mimas has thicker thermal lithosphere than Enceladus, by using a one-dimensional

142 simulation of thermal evolution under various conditions. In section 2, we describe the

143 interior structure models we used, the details of the thermal evolution model, and the 
144 parameter settings. In section 3, we show the results. Changes in the thickness of the

145 shell and ocean with time and the dependence of the final ocean and lithosphere

146 thicknesses on the parameters are shown. In section 4, we discuss the relevance between

147 our results and other observations and simulations. In section 5, we offer conclusions.

\section{2. Methods}

\section{$149 \quad 2.1$ Interior structure model}

150 We consider Enceladus and Mimas to be 4-layered spheres of rock - ocean - methane

151 hydrate - ice. This layered structure is assumed to be achieved initially by early

152 differentiation caused by the energies of decay of short-lived radioactive elements and

153 accretion energy (Schubert et al. (2007)). The interior structure models are shown in

154 Figure 1, and the model parameters are summarized in Table 2. The size of the rocky

155 cores was calculated using the ice shell thickness and density currently estimated from

156 observations. The ice thickness of Enceladus is assumed to be $20 \mathrm{~km}$, the ice shell

157 thickness of Mimas is assumed to be $30 \mathrm{~km}$, and the density of the rocky cores is

158 assumed to be $2450 \mathrm{~kg} \mathrm{~m}^{-3}$. Other values are given in Table 3 . Then, we obtained the

159 size of the Enceladus rocky core is $190.7 \mathrm{~km}$, and the size of the Mimas rocky core is 
$98.9 \mathrm{~km}$. These values are consistent with the values estimated from observation data of

161 Enceladus and Mimas (e.g., Tajeddine et al. (2014)). In the ocean, we assume that only

162 methane has dissolved. We do not consider the presence of ammonia because the

163 amount of ammonia observed by Cassini $(\sim 1 \%)$ would not notably affect the ice

164 freezing point (Choukroun and Grasset 2010; Vu et al. 2014). It is assumed that the

165 composition formula of methane hydrate is $\mathrm{CH}_{4} \bullet 6 \mathrm{H}_{2} \mathrm{O}$, and its density is $920 \mathrm{~kg} \mathrm{~m}^{-3}$.

$166 \quad 2.2$ Thermal evolution

$167 \quad$ 2.2.1 Heat transfer

168 To calculate the evolution of heat transport with time, including conduction and

169 convection, the modified mixing length theory, the one-dimensional calculation scheme

170 developed by (Kamata (2018)), is used. The governing equation of heat transfer is given

171 by

172

$$
\rho C_{p} \frac{\mathrm{d} T}{\mathrm{~d} t}=-\frac{1}{r^{2}} \frac{\mathrm{d}}{\mathrm{d} r}\left(r^{2} F_{\text {cond }}+r^{2} F_{\text {conv }}\right)+Q
$$

173 where $\rho$ is the density, $C_{p}$ is the specific heat, $T$ is the temperature, $r$ is the radius,

$174 F_{\text {cond }}$ is the conductive heat flux, $F_{\text {conv }}$ is the convective heat flux, and $Q$ is the

175 volumetric heating rate. The thermal conductivity and specific heat of each layer are 
given in Table 2. $F_{\text {cond }}$ and $F_{\text {conv }}$ are given by

$$
\begin{aligned}
& F_{\text {cond }}=-k \frac{\mathrm{d} T}{\mathrm{~d} r} \\
& F_{\mathrm{conv}}=-k_{v}\left\{\frac{\mathrm{d} T}{\mathrm{~d} r}-\left(\frac{\mathrm{d} T}{\mathrm{~d} r}\right)_{s}\right\}
\end{aligned}
$$

178 where $k$ is the thermal conductivity, $k_{v}$ is the effective thermal conductivity, and

$179(\mathrm{~d} T / \mathrm{d} r)_{s}$ is the adiabatic temperature gradient, that is $-\alpha g T / C_{p} . \alpha$ is the thermal

180 expansivity. $k_{v}$ is given by

$$
k_{v}=\left\{\begin{aligned}
-\frac{\alpha C_{p} \rho^{2} g l^{4}}{18 \eta}\left\{\frac{\mathrm{d} T}{\mathrm{~d} r}-\left(\frac{\mathrm{d} T}{\mathrm{~d} r}\right)_{s}\right\}, & \left(\frac{\mathrm{d} T}{\mathrm{~d} r} \leq\left(\frac{\mathrm{d} T}{\mathrm{~d} r}\right)_{s}\right) \\
0, & \left(\frac{\mathrm{d} T}{\mathrm{~d} r}>\left(\frac{\mathrm{d} T}{\mathrm{~d} r}\right)_{s}\right)
\end{aligned}\right.
$$

182 where $g$ is the gravitational acceleration, $\eta$ is the viscosity, and $l$ is the mixing

183 length. $l$ depends on the computational grid temperature, shell thickness, and other

184 parameters (Kamata (2018)). Gravitational acceleration is determined from the equation

185 of hydrostatic equilibrium. The viscosities of ice and methane hydrate are as follows:

$$
\eta(T)=\eta_{\mathrm{ref}} \exp \left(\frac{E_{a}}{R_{g} T_{\mathrm{ref}}}\left(\frac{T_{\mathrm{ref}}}{T}-1\right)\right)
$$

187 where $\eta_{\text {ref }}$ is the reference viscosity and corresponds to the viscosity at $T_{\text {ref }}, T_{\text {ref }}$ is

188 the melting point of ice or the dissociation point of methane hydrate, $E_{a}$ is the

189 activation energy, and $R_{g}$ is the gas constant (e.g., Goldsby and Kohlstedt (2001)). We

190 assume that the reference viscosity of ice is $10^{14} \mathrm{Pas}$ and that of methane hydrate is 
$1912.0 \times 10^{15}$ Pa s (Kamata et al. 2019; Durham et al. 2003). These reference viscosities

192 have uncertainties since these values depend on the porosity and grain sizes of the icy

193 shell, and they are unknown. Solid convection in the rocky core is not considered, i.e.,

$194 F_{\text {conv }}$ is 0 in the rocky core. We assume that heat received from the rocky core transfers

195 to the bottom of the icy shell immediately in the ocean, and the temperature remains

196 constant at the freezing point of the shell bottom. The adiabatic gradient in the ocean is

197 ignored since the gradient is small. Heat sources are the decay of long-lived radioactive

198 elements included in the rocky core and tidal heat in solid parts. The rocky core is

199 assumed to be carbonaceous chondrites. The amounts of long-lived radioactive elements

200 are summarized in Table 3 (Lodders (2003)). The details of the tidal heating rate are

201 described in a later section.

\section{$202 \quad$ 2.2.2 Shell growth}

203 Icy shell growth is described by the energy balance at the shell/ocean boundary. If the

204 temperature at the shell bottom exceeds the melting point, differences between

205 incoming heat flux and outgoing heat flux will melt the icy shell. The change in the

206 shell thickness is given by 


$$
\rho_{\text {ice }} L_{\text {eff }} \frac{\mathrm{d} D}{\mathrm{~d} t}=F_{\text {out }}-F_{\text {in }}
$$

208 where $D$ is the icy shell thickness, $L_{\text {eff }}$ is the effective latent heat, $F_{\text {out }}$ is the heat

209 flux outgoing from the shell bottom, and $F_{\text {in }}$ is the heat flux incoming into the shell

210 bottom. $L_{\text {eff }}$ is the sum of the latent heat of the shell and the heat used for warming the

211 ocean to the melting point at the shell bottom. This value is given by

$$
L_{\text {eff }}=L-\frac{\rho_{\text {oce }} g_{b o t} C_{p, \text { oce }} V_{\text {oce }}}{4 \pi R_{\text {bot }}^{2}} \frac{\mathrm{d} T_{\mathrm{m}}}{\mathrm{d} P_{\text {bot }}}
$$

213 where $L$ is the latent heat, $V_{\text {oce }}$ is the volume of the ocean, and $R_{\text {bot }}$ is the radius at

214 the bottom of the layer. $C_{p, \text { oce }}$ is the specific heat of pure water, which depends on

215 temperature (Choukroun and Grasset (2010)), and is given by

$$
C_{p, \text { oce }}=4190+9 \exp \left(-0.11\left(T_{\text {oce }}-281.6\right)\right)
$$

217 where $T_{\text {oce }}$ is the ocean temperature. The melting point of ice $T_{\mathrm{m}, \mathrm{ice}}$ depends on

218 pressure, and is given by

$$
T_{\mathrm{m}, \mathrm{ice}}(P)=273.1-c_{1} P-c_{2} P^{2}
$$

220 where $P$ is the pressure, $c_{1}=7.95 \times 10^{-8} \mathrm{~K} \mathrm{~Pa}^{-1}$, and $c_{2}=9.6 \times 10^{-17} \mathrm{~K} \mathrm{~Pa}^{-2}$

221 (Leliwa-Kopystyński et al. (2002)). The dissociation point of methane hydrate is given 


$$
T_{\text {m,hyd }}=\frac{b}{\ln \left(\frac{P}{1000}\right)-a}
$$

224 where $a=38.980$ and $b=-8533.80 \mathrm{~K}$ (Sloan Jr and Koh (2007)). The growth of

225 the methane hydrate layer is slightly different from that of the ice layer. We adopt a

226 method for methane hydrate layer growth similar to that of Kamata et al. (2019) and

227 summarize it below. The methane hydrate layer grows thicker if the outgoing heat flux

228 is higher than the incoming heat flux. The temperature of the ocean remains constant at

229 that time. If the incoming heat flux is higher than the outgoing heat flux, the ocean

230 temperature increases until it reaches the dissociation point. After the ocean temperature

231 exceeds the dissociation point of methane hydrate, the hydrate layer starts to dissociate.

232 We change the method slightly to introduce methane concentrations into the ocean. In

233 our calculation, methane hydrate can form when methane is supersaturated in the ocean

234 and the dissociation point of methane hydrate is lower than that of ice. The methane

235 solubility at the ocean/shell boundary, which is at methane gas - water - methane

236 hydrate equilibrium, can be determined from the temperature or the pressure (Duan and

237 Mao (2006)). We note a fitting curve that reproduces the methane solubility in table 14

238 in (Duan and Mao (2006)), which is given by 


$$
m_{\mathrm{CH}_{4}, \text { satu,bou }}=\exp \left(A\left(\frac{1}{T_{\text {oce }}}\right)^{3}+B\left(\frac{1}{T_{\text {oce }}}\right)^{2}+C\left(\frac{1}{T_{\text {oce }}}\right)+D\right)
$$

240 where $T_{\text {oce }}$ is the ocean temperature, $m_{\mathrm{CH}_{4} \text {,satu,bou }}$ is the methane solubility at the

241 ocean/shell boundary and its unit is $\mathrm{mol} \mathrm{m}^{-3}, A=9.1589 \times 10^{9} \mathrm{~K}^{3} \mathrm{~mol} \mathrm{~m}^{-3}, B=$

$242-9.7261 \times 10^{7} \mathrm{~K}^{2} \mathrm{~mol} \mathrm{~m}^{-3}, C=3.3963 \times 10^{5} \mathrm{~K} \mathrm{~mol} \mathrm{~m}^{-3}$, and $D=$

$243-3.8513 \times 10^{2} \mathrm{~mol} \mathrm{~m}^{-3}$. When methane hydrate dissociates or forms, the methane

244 concentration in the ocean changes. Let the methane concentration in the ocean be

$245 C_{\mathrm{CH}_{4}, \text { oce }}$ and the change in methane concentration be $\Delta C_{\mathrm{CH}_{4}, \mathrm{oce}} \cdot \Delta C_{\mathrm{CH}_{4}, \text { oce }}$ is given by

$$
\Delta C_{\mathrm{CH}_{4}, \mathrm{oce}}=\frac{C_{\mathrm{CH}_{4}, \mathrm{oce}} V_{\text {oce }}-C_{\mathrm{CH}_{4}, \mathrm{MH}} \Delta V_{\mathrm{MH}}}{V_{\text {oce }}-\Delta V_{\text {oce }}}-C_{\mathrm{CH}_{4}, \text { oce }}
$$

247 where $V_{\text {oce }}$ is the ocean volume, $\Delta V_{\text {oce }}$ is the change in the ocean volume, $\Delta V_{\mathrm{MH}}$ is

248 the change in the methane hydrate layer volume, and $C_{\mathrm{CH}_{4}, \mathrm{MH}}$ is the methane

249 concentration included in methane hydrate. Since the composition formula of methane

250 hydrate is $\mathrm{CH}_{4} \cdot 6 \mathrm{H}_{2} \mathrm{O}$ and its density is $920 \mathrm{~kg} \mathrm{~m}^{-3}, C_{\mathrm{CH}_{4}, \mathrm{MH}}$ is $7419 \mathrm{~mol} \mathrm{~m}^{-3}$.

251 Once the methane concentration falls below the soluble concentration by methane

252 hydrate formation, the dissociation points of methane hydrate decreases (Choukroun et

253 al., 2010). Even if ocean is methane unsaturated, if the dissociation point of methane

254 hydrate is higher than the melting point of ice, methane hydrate layer will grow. 
255 However, when the methane in the ocean is less than $0.1 \%$, the dissociation point of

256 methane hydrate is about $40 \mathrm{~K}$ lower than that of the case where ocean is supersaturated

257 with methane. When methane hydrate is in equilibrium with water and methane gas, the

258 methane solubility is about $0.11 \%$ under the conditions of $275.15 \mathrm{~K}$ and $3.2 \mathrm{MPa}$ (Duan

259 and Mao (2006)). Therefore, the growth of methane hydrate alone in the methane

260 unsaturated ocean is ignored in this study. Instead, we introduced a mix layer in which

261 methane hydrate and ice grow simultaneously when the ocean becomes methane

262 unsaturated, and the mix layer begins to form when the ocean becomes methane

263 unsaturated and the ocean temperature becomes equal to the melting point of ice at that

264 depth. The properties of the mixture are a linear combination of methane hydrate and

265 ice, with the exception of viscosity., which is given by

$$
A_{\text {mix }}=\phi_{\text {hyd }} A_{\text {hyd }}+\left(1-\phi_{\text {hyd }}\right) A_{\text {ice }}
$$

267 where $A$ represents a material property and $\phi_{\text {hyd }}$ is the volume fraction of methane

268 hydrate to ice in a calculated cell. The viscosity of the mixture is given by

$$
\eta_{\text {mix }}=\eta_{\text {hyd }}^{\phi_{\text {hyd }}} \eta_{\text {ice }}^{\left(1-\phi_{\text {hyd }}\right)}
$$

\subsubsection{Calculation}


271 Our code can reproduce the results for Kamata et al. (2019), Kamata (2018), and

272 Kimura and Kamata (2020). The icy shell is divided into 100 thin layers. The maximum

273 thickness of the layer is $\sim 1 \mathrm{~km}$ when there is no ocean in Mimas. The rocky core is also

274 divided into 100 thin layers. The time step is chosen to satisfy the Courant-Friedrichs-

275 Lewy (CFL) condition. The calculation is finished when the sum of the time steps

276 exceeds 4.5 Gyr.

\section{$277 \quad 2.3$ Initial conditions and parameter settings}

\section{$278 \quad$ 2.3.1 Initial structure and temperature}

279 Our calculation starts after differentiation, which finishes within approximately 100

280 Myr after CAI formation (Schubert et al. (2007)). Differentiation was caused by heat

281 derived from short-lived radioactive species such as ${ }^{26} \mathrm{Al}$ and accretion energy. During

282 differentiation of Enceladus, its core temperature reached $\sim 1273 \mathrm{~K}$ (Schubert et al.

283 (2007)). Mimas is also differentiated, but its core temperature is not heated as much

284 (Neveu and Rhoden (2017)). We believe the icy shell must have been melted initially,

285 and the liquid layer survives for the existence of the ocean because tidal dissipation

286 does not effectively occur once the ocean completely freezes (Roberts and Nimmo 
287 (2008)). The highest temperature that the rocky core experienced during differentiation

288 depends on the amount of ${ }^{26} \mathrm{Al}$ in the core when the moon accreted. However,

289 determining when the moons formed and how much ${ }^{26} \mathrm{Al}$ is in the core is complicated.

290 Here, we consider the initial isothermal core temperature to be a free parameter in a

291 range between $273 \mathrm{~K}$ and $1273 \mathrm{~K}$. The nominal values are $1273 \mathrm{~K}$ for Enceladus and

$292573 \mathrm{~K}$ for Mimas. The thickness of the initial icy shell is set to $10 \mathrm{~km}$ and the

293 temperature has a linear gradient from the surface to the ocean. The initial shell does not

294 include any methane hydrate. The surface temperature is set to a constant value

295 described in Table 1. The initial temperature of the ocean is set to $273 \mathrm{~K}$. The

296 differences in the initial structures except for completely frozen state does not

297 considerably affect the final structures. Even at the size of Pluto, the initial internal

298 structure has little effect on the final internal structure (Kimura and Kamata (2020)).

$299 \quad$ 2.3.2 Tidal heating rate

300 In previous studies, tidal heating rates on Saturnian moons were estimated (e.g., Meyer

301 and Wisdom (2007)). However, determining the history of tidal heating rates is difficult

302 because the heating rates strongly depend on the interior structure and orbital properties, 
303 and their evolution is quite complicated. Tidal heating which depends on the viscosity

304 structure inside the icy shell, has been proposed (Tobie et al., (2003)), but the modified

305 mixing length theory cannot handle the heat generated inside the icy shell. Therefore,

306 we adopt an assumption similar to Kamata (2018), where the constant tidal heating rate

307 is added at the bottom of the icy shell. In our calculation, we consider the average tidal

308 heating rate as a free parameter in a range between 0 and $30 \mathrm{GW}$ for simplicity. The

309 tidal heat is assumed to be generated in solid parts of the moons, namely, the rocky core

310 and icy shell. We do not consider dissipation in the ocean because its value is small

311 enough to ignore (Chen et al. (2014)). In a rocky core, the amount of tidal dissipation

312 depends on the properties of the rocky core. If the core is rigid, dissipation does not

313 effectively occur due to its high viscosity (Roberts and Nimmo (2008)). Porous rocky

314 cores such as gravel dissipate well, and high heat flow is generated (Choblet et al.

315 (2017)). In an icy shell, the dissipation depends on its viscosity. Tidal heat is produced

316 most effectively when the shell is near the melting point (Tobie et al. (2003)); i.e., tidal

317 heat is generated most at the shell bottom. Souček et al. (2019), which investigated the

318 effect of variations in ice shell thickness on tidal deformation in Enceladus, found that 
319 tidal heating inside the ice shell is less than $1 \mathrm{GW}$, except near the south pole.

320 Therefore, it is not completely clear where and how much tidal heating is occurring in

321 the solid parts of Enceladus and Mimas. We assume that all of the tidal heating is used

322 at the base of the ice shell to melt the shell and increase ocean temperature. The average

323 tidal heat in our calculation represents the sum of the tidal heat generated in the icy shell

324 and the rocky core. Thus, $F_{\text {in }}$ is described by

$$
4 \pi R_{\mathrm{bot}}^{2} F_{\mathrm{in}}=4 \pi R_{\mathrm{c}}^{2} F_{\mathrm{c}}+Q_{t}
$$

326 where $R_{\mathrm{c}}$ is the radius of the rocky core, $F_{\mathrm{c}}$ is the heat flux at the surface of the rocky

327 core, and $Q_{t}$ is the average tidal heating rate. Since tidal heating becomes ineffective

328 once the ocean freezes completely (Roberts and Nimmo (2008)), the tidal heating rate is

329 set to 0 if there is no ocean. In our model, we assume that all tidal heating is used to

330 melt the icy shell and increase the ocean temperature. Numerical experiment for the

331 convection of the ice shell suggests that the tidal heating occurs most at the bottom of

332 the icy shell (Tobie et al., (2003)), and recent numerical model for the tidal deformation

333 of Enceladus' icy shell indicates that the tidal dissipation in the icy shell is small

334 (Souček et al. (2019)). Nevertheless, tidal heating warms the lower part of the icy shell 
336 of a well-convected icy shell, our assumption is an oversimplification. If tidal heating

337 were also used to increase the temperature of the icy shell, then convection in the icy

338 shell would be promoted. As a result, the icy shell would be cooled more efficiently and

339 the final ocean thickness would be thinner than the thickness determined in this study.

\section{$340 \quad$ 2.3.3 Initial methane concentration}

341 The rocky core needs to be heated to approximately $300{ }^{\circ} \mathrm{C}$ so that methane is formed

342 by the serpentinization and reduction of carbon dioxide (McCollom (2016)). However,

343 heat only from long-lived radioactive decay cannot sufficiently warm the core due to the

344 small-sized cores of Enceladus and Mimas. Therefore, we assume that methane is

345 produced during early differentiation by the heat derived from decay of short-lived

346 radioactive elements and/or that methane existed initially in the moons. The amount of

347 methane produced by the serpentinization of the rocky core depends on the initial

348 amount of olivine in the core and the degree of hydration of the core. Comets

349 considered to be parts of icy moons contain $\sim 1 \% \mathrm{CH}_{4}$ relative to water (Mumma and

350 Charnley (2011)). This value is approximately equivalent to $\sim 600 \mathrm{~mol} \mathrm{~m}^{-3}$ methane. 
351 As the amount of methane in an initial subsurface ocean depends on a complicated

352 condition at the accretion of the moons and it is unknown, this amount is designated a

353 free parameter in a range of $0-1000 \mathrm{~mol} \mathrm{~m}^{-3}$. When the ocean temperature is $273.15 \mathrm{~K}$

354 and the pressure is $2.6 \mathrm{MPa}$, the solubility of methane is $\sim 60 \mathrm{~mol} \mathrm{~m}^{-3}$. The previous

355 study which considered methane hydrate layer in Pluto similarly assumed that methane

356 is supersaturated in the ocean (Kamata et al. (2019)). Supplementary Figure 7 in

357 Kamata et al. (2019) shows that $\sim 1 \% \mathrm{CH}_{4}$ allows $>10 \mathrm{~km}$ methane hydrate layer to

358 form in the subsurface ocean of Pluto. For another source of methane, ongoing methane

359 production may be considered. If tidal dissipation occurs in the rocky core, methane

360 could possibly be produced by tidal heat. Additionally, it is known that methanogen can

361 survive under the conditions present in the ocean of Enceladus (Taubner et al. (2018)),

362 and methane may be produced by them now. To simplify our calculation, we do not

363 consider such ongoing methane production.

3643 Results

$365 \quad 3.1$ Change in shell thickness with time

366 Figure 2 shows one of the results of the icy shell thickness change with time for Mimas. 
367 If a methane hydrate layer exists beneath the pure ice layer, the final ocean is thicker

368 than that in the case without methane hydrate layer under the same tidal heating rate and

369 initial core temperature. This is because the viscosity of the methane hydrate layer is

370 higher than that of ice, and the convection is depressed as shown in the formula of $k_{v}$.

371 The methane hydrate layer prevents the icy shell from convecting and insulates the heat

372 from the bottom of the shell or deeper region. As a consequence of the insulation of the

373 methane hydrate layer, the ice layer over the methane hydrate layer becomes cooler,

374 which leads to a higher viscosity of ice. Hence, the icy shell convection in the case with

375 methane hydrate is depressed and $F_{\text {conv }}$ is nearly 0 in all areas of the shell.

376 The current shell thickness is achieved in the early stage of evolution. This is because

377 the energy of radioactive decay is much lower than tidal heating due to the small rocky

378 core. The thicknesses of the icy shells are controlled by both the radiogenic heating the

379 tidal heating. Because of the small size of both Enceladus and Mimas, the tidal heat at

380 the base of the icy shell immediately reaches a near equilibrium with the outgoing heat.

381 In fact, tidal heat is not constant with time, as it strongly depends on orbital evolution

382 and structural evolution of the interior. Therefore, the evolution of the internal structure 
383 shown in Fig. 2 does not account for variations in tidal heating. However, as mentioned

384 above, the internal structure almost reaches equilibrium in $100 \mathrm{Myr}$, so the final internal

385 structure shown in Figure 2 can be achieved if the average tidal heating over the last

$386100 \mathrm{Myr}$ is close to constant.

387 The core temperature evolution of Mimas was also investigated. However, the core

388 temperature rapidly decreases to near the ocean temperature without tidal heating. In

389 addition, the effect of the differences in the initial core temperature on the final shell

390 thickness is investigated. The shell thicknesses for each evolution which starts from

391 different initial core temperature attain a similar value in approximately $100 \mathrm{Myr}$, and

392 the final shell thicknesses of all initial core temperatures achieve the same value. It is

393 found that the final shell thickness does not depend on the initial core temperature, in

394 other words, the heat stored initially in the rocky core is much lower than the heat added

395 into the icy shell, namely the tidal heat and the heat generated by the decay of the

396 radioactive elements. Similar tendencies are also found in the results for Enceladus.

397 Therefore, in our calculation, the typical initial temperatures are chosen $573 \mathrm{~K}$ for

398 Mimas and $1273 \mathrm{~K}$ for Enceladus. These temperatures are consistent with the core 
400 considering the heat from the decay of short-lived radioactive elements was calculated

401 (Schubert et al. 2007; Neveu and Rhoden 2017).

\section{$402 \quad 3.2$ The dependency of final shell thickness on initial methane concentration}

403 Figure 3 shows the differences in final structures under different initial methane

404 concentrations in the ocean and $3 \mathrm{GW}$ for the tidal heating rate for Mimas. As the initial

405 methane concentration decreases, the final ocean thickness decreases. If the initial

406 methane concentration is less than $500 \mathrm{~mol} \mathrm{~m}^{-3}$, the mixed layer of ice and methane

407 hydrate is laid beneath the pure methane hydrate layer. The properties of the mixed

408 layer are similar to those of ice, and therefore, the mixed layer cannot insulate as much

409 incoming heat as the methane hydrate layer. This is because the initial subsurface ocean

410 is not supersaturated by methane, and the pure methane hydrate layer cannot form.

411 Under the condition that the initial concentration is more than $500 \mathrm{~mol} \mathrm{~m}^{-3}$, the final

412 ocean thicknesses under any methane concentration become the same value since the

413 ocean is supersaturated during growth, and the mixed layer does not appear. In our

414 calculation, the typical values are $1000 \mathrm{~mol} \mathrm{~m}^{-3}$ for the case with the methane hydrate 
415 layer and $0 \mathrm{~mol} \mathrm{~m}^{-3}$ for the case without the methane hydrate layer.

$416 \quad 3.3$ Differences in final shell thickness and lithosphere among different tidal

\section{7 heating rates}

418 Figure 4 shows the final shell and ocean thickness and the thermal lithosphere of

419 Enceladus and Mimas under different tidal heating rates. The thermal lithosphere

420 denotes the nonconvective area in the shell where $F_{\text {conv }}$ is 0 . If the total dissipated

421 power is the same for Enceladus and Mimas, then the heating rate per unit mass for

422 Mimas is higher because it is smaller. In the regions between 2.0 GW and 5.0 GW for

423 Figure 4(a) and between $7.5 \mathrm{GW}$ and $20 \mathrm{GW}$ for Figure 4(c), the final shell thickness is

424 a plateau, where the methane hydrate layer insulates the shell from the inner heat and

425 the convective area in the shell is thin; in other words, the thermal lithosphere is thick.

426 If the methane hydrate layer exists in an icy shell, a thermal lithosphere is thicker than

427 that of the icy shell without the methane hydrate layer. This is attributed to methane

428 hydrate insulation. If the tidal heating rate for Enceladus is more than $20 \mathrm{GW}$ and the

429 tidal heating rate for Mimas is more than 7.5 GW, no methane hydrate layers appear

430 inside the icy shell. This means that there is no region where the dissociation point of 
431 methane hydrate is higher than the melting point of ice because the entire icy shell is at

432 low pressure.

\section{$433 \quad$ 3.4 Surface heat flux of Mimas}

434 Figure 5 shows the current surface heat flux versus the final shell thickness of Mimas.

435 In the region where the final shell thickness is more than $\sim 40 \mathrm{~km}$ in the figure, the

436 surface heat flux of the case with methane hydrate is depressed more than that of the

437 case without methane hydrate due to methane hydrate insulation. When the thickness of

438 the icy shell is smaller than $30 \mathrm{~km}$, the surface heat fluxes are equal for the case with

439 and without in the ocean. This is because, as mentioned in the previous subsection, if

440 the icy shell is too thin, methane hydrate layers do not appear. The low surface heat flux

441 on Mimas may contribute to the maintenance of surface topography on Mimas. Details

442 are discussed in the discussion section.

\section{$443 \quad 3.5$ Summary of results}

444 In Figure 6, the results from Figure 4 are summarized, and the ranges in which a thick

445 subsurface ocean can exist in both moons and where the current thermal lithosphere of

446 Mimas is thicker than that of Enceladus are shown. For the thick ocean criteria, 
447 thicknesses greater than $50 \mathrm{~km}$ for Mimas and greater than $25 \mathrm{~km}$ for Enceladus are

448 assumed. Since the ocean thicknesses of both moons are not completely known, we

449 assumed that the criteria are about half of the $\mathrm{H}_{2} \mathrm{O}$ layer (including the icy shell and the

450 ocean). The area that is colored red and surrounded by the dashed line in the figure is

451 the preferred region where the thick subsurface ocean exists and the thermal lithosphere

452 of Mimas is thicker than that of Enceladus. To achieve a thick ocean in these bodies,

453 Mimas needs $\sim 2.0 \mathrm{GW}$ and Enceladus needs $\sim 7.5 \mathrm{GW}$ of tidal heating rate in the case

454 of methane hydrate, while Mimas needs $\sim 5.0 \mathrm{GW}$ and Enceladus needs $\sim 15 \mathrm{GW}$ in

455 the case without methane hydrate. The preferred region for the case with methane

456 hydrate is larger than that for the case without methane hydrate, which means that the

457 current states of Mimas and Enceladus are achieved more easily if a methane hydrate

458 layer exists beneath the ice layer.

459 4. Discussion

$460 \quad 4.1$ The dependence of tidal dissipation factor $\boldsymbol{Q}_{\mathbf{p}}$

461 The thermal evolution of the moons is closely associated with their orbital evolution

462 (Nimmo et al. (2018)). This is because the tidal heating rate, which controls the thermal 
463 evolution of Mimas and Enceladus, depends not only on their internal structures but

464 also on their orbits. For moons with MMR, the equilibrium tidal heating rate can be

465 estimated as shown in equations (2) and (3). The tidal dissipation factor of the primary

$466 Q_{\mathrm{p}}$ in the equations influences the tidal heating rate in the moons and the orbital

467 evolution of the moons. An astrometric study estimated that the $Q_{\mathrm{p}}$ of Saturn is $\sim$

4682000 (Lainey et al. (2012)). If $Q_{\mathrm{p}} \sim 2000$ was constant, Mimas and Enceladus were

469 possibly formed more recently (e.g., Charnoz et al. (2011)). As shown in Figure 2, it is

470 found that the current interior of Mimas was achieved in $100 \mathrm{Myr}$ from the initial state,

471 and the interior structure can be achieved even though Mimas formed 100 Myr ago. The

472 same finding applies to Enceladus whose size is slightly larger than that of Mimas.

473 Note, however, that we do not consider differentiation, failure, and the relaxation of

474 craters in the calculations.

475 Fuller et al. (2016) proposed that $Q_{\mathrm{p}}$ does not have to be constant and that the value

476 used to be higher; this was called the resonance locking hypothesis. Under this

477 hypothesis, Mimas and Enceladus could have formed 4.6 Gyr ago and Mimas went

478 through 3:2 Mimas-Enceladus MMR 0.34 Gyr ago when $Q_{\mathrm{p}}$ was $\sim 4800$, and the 
equilibrium tidal heating rate for Mimas, following equations (2) and (3), was 3.6

480 GW (Tian and Nimmo (2019)). The tidal heating rate can thicken the subsurface ocean

481 in Mimas if the methane hydrate layer exists. Now, Enceladus is in the Enceladus-Dione

482 MMR and the equilibrium tidal heating rate is $\sim 10 \mathrm{GW}$. The tidal heating rate can

483 thicken the subsurface ocean in Enceladus if the methane hydrate layer exists.

484 Assuming constant tidal heating, we found that Enceladus and Mimas reach near

485 equilibrium within about $100 \mathrm{Myr}$. If tidal heating of $10 \mathrm{GW}$ for Enceladus and $3.6 \mathrm{GW}$

486 for Mimas had been added for about last 100 Myr, a thick subsurface ocean would be

487 realized in both satellites if methane hydrate is present. Observational data are used to

488 estimate the current shell thicknesses of both bodies. The estimated shell thickness of

489 Enceladus ranges between $14 \mathrm{~km}$ and $50 \mathrm{~km}$, which means that the subsurface ocean is

490 at least $10 \mathrm{~km}$ (e.g., Iess et al. (2014)). Figure 6 shows that $10 \mathrm{GW}$ of tidal heating in

491 Enceladus can be achieved with ice alone for $10 \mathrm{~km}$ of ocean, but methane hydrate is

492 needed to achieve thicker ocean of $25 \mathrm{~km}$ or more. Libration data are used to estimate

493 the shell thickness of Mimas: the thickness ranges between $24 \mathrm{~km}$ and $31 \mathrm{~km}$

494 (Tajeddine et al. (2014)), which corresponds to the ocean thickness between $68.3 \mathrm{~km}$ 
496 for Mimas is sufficient to maintain $50 \mathrm{~km}$ of ocean in the presence of methane hydrate

497 layer, but not sufficient to maintain the ocean thickness between $68.3 \mathrm{~km}$ and $75.3 \mathrm{~km}$.

498 This may be explained by the presence of dissolved gases other than methane in the

499 ocean. Dissolved gas, which can be encapsulated in gas hydrate cages, may affect the

500 final ocean thickness. The stable pressure for mixed hydrates of methane and other

501 gaseous species is different from that for pure methane hydrate, which changes the

502 ice/gas hydrate boundary depth and the final ocean thickness. Details of the dissolved

503 gaseous species in the ocean are discussed in a later subsection.

\section{$504 \quad 4.2$ Relevance to geophysical features}

505 Mimas has few tectonic features on its surface (Rhoden et al. (2017)). This is a large

506 constraint for the thermal evolution of Mimas. Rhoden et al. (2017) estimated the tidal

507 stress on the icy shell of Mimas when the subsurface ocean existed. The tidal stress is

508 over $1300 \mathrm{kPa}$ when the viscosity of the shell of the lower parts is $10^{12} \mathrm{~Pa}$, which

509 leads to the shell failure. In order to reduce the tidal stress, the viscosity of the icy shell

510 must be high, i.e. the temperature of the icy shell must be low. The existence of a 
511 methane hydrate layer may offer an advantage in that the icy shell remains cool due to

512 the insulation of methane hydrate. Thermal stress derived from phase change may also

513 cause shell failure. To avoid thermal stress, there must be no ocean or the changes in

514 ocean thickness must be much slower. As shown in Figure 2, the changes in ocean

515 thickness with time under a constant tidal heating rate is little except for the initial 100

516 Myr. Hence, the effects of change in the tidal heating rate on the shell thickness is more

517 significant. As shown in Figure 4(a) and 4(c), the final shell thickness changes little in

518 the regions where methane hydrate exists (i.e., 2.0-5.0 GW for Mimas and 7.5-20 GW

519 for Enceladus), which means that the change in shell thickness is insensitive to the

520 change in tidal heating rate under the condition that a methane hydrate layer exists.

521 Methane hydrate layer suppresses the changes in the shell thickness. Note, however,

522 that the tidal heating rate is closely associated with orbital evolution; the details must be

523 investigated by another study.

524 In Tian and Nimmo (2019), the surface heat flux on Mimas is estimated to be less than

$52510 \mathrm{~mW} / \mathrm{m}^{2}$ from the condition of the largest crater Herschell, which is not notably

526 relaxed. As shown in Figure 5, the heat flux is depressed sufficiently if the methane 
527 hydrate layer exists. The surface heat flux is mainly determined by the total heat

528 retained in the interior. As shown in Figure 4, if the same tidal heating rate is applied to

529 the icy bodies with and without methane in the ocean, the final ocean thickness is

530 thicker in the case with methane in the ocean. Therefore, when methane hydrate layers

531 are present in the icy shell, both a thin ice shell and low surface heat flux can be

532 achieved. As mentioned above, the visual appearance of Mimas may be explained by

533 assuming a methane hydrate layer beneath the icy shell.

534 Craters on Enceladus are highly relaxed, and the surface is thought to experience high

535 heat flux. The high heat flow is supposed to be derived from the regolith layer near the

536 surface, which originates from the plume and E-ring (Bland et al. (2012)). The regolith

537 layer has high porosity, and relatively low thermal conductivity, which plays a role in

538 insulation like the methane hydrate layer. The regolith layer creates a high-temperature

539 gradient near the surface. The high-temperature gradient results in viscous relaxation of

540 the craters on Enceladus surface. Therefore, the presence of relaxed craters may be

541 independent of whether the methane hydrate layer exists, and it is difficult to discuss

542 any relevance between the geophysical features on the Enceladus surface and methane 
543 hydrate existence. Although it is difficult to consider a local geophysical feature

544 because our calculation has only one dimension, it may be unlikely that methane

545 hydrate or any other hydrate would form at the south polar terrain since the shell

546 thickness is thinner there than at any other region of Enceladus (Čadek et al. (2016)),

547 and the dissociation points of gas hydrates are lower than that of ice. Hence, the

548 viscosity of a shell at the south polar terrain that does not include methane hydrate may

549 be lower than that at the other region.

$550 \quad 4.3$ Dissolved gaseous species

551 Hydrate cages can incorporate not only methane but also other gases dissolved in the

552 ocean. Components observed in the Enceladus plume would form mixed hydrates under

553 pressure and temperature in the ocean (Bouquet et al. (2015)). Pure methane hydrate

554 and mixed hydrate have different properties such as dissociation point and density.

555 Because of the differences in dissociation points, the final ocean thickness may change

556 because the depth at which gas hydrates appear may change. The difference in density

557 affects whether the gas hydrate layer can exist beneath the ice layer. The density of

558 mixed hydrates depends on the structure of the hydrates and the involved gaseous 
559 species. If gas hydrates form from the Enceladus plume composition, two types of

560 hydrates can exist: structure I mixed hydrates, with a density of $1040 \mathrm{~kg} \mathrm{~m}^{-3}$, and

561 structure II mixed hydrates, with a density of $970 \mathrm{~kg} \mathrm{~m}^{-3}$ (Bouquet et al. (2015)).

562 Structure II mixed hydrates can ascend in an ocean, but whether structure I mixed

563 hydrates ascend in the ocean depends on the density of the ocean. The ascended

564 hydrates can exist beneath the icy shell, while the sunken hydrates may be dissolved by

565 heat from the rocky core. $\mathrm{CO}_{2}$ is important in the dissolved gaseous species since $\mathrm{CO}_{2}$ is

566 heavier than $\mathrm{CH}_{4}$ and $\mathrm{CO}_{2}$ coexists with $\mathrm{CH}_{4}$. The ratio of $\mathrm{CO}_{2}$ to $\mathrm{CH}_{4}$ in the ocean

567 depends on the temperature near the core. $\mathrm{CO}_{2}$ dominates in the ocean at over $450{ }^{\circ} \mathrm{C}$

568 (McCollom and Seewald (2007)). If the temperature of the rocky core is high, carbon

569 dioxide dominate in the ocean and gas hydrates may not form. However, the

570 temperature of the core is influenced by how much tidal dissipation occurs in the core,

571 which is beyond our focus. We assume that the initial methane concentration would be

$5721000 \mathrm{~mol} \mathrm{~m}^{-3}$ for most of the cases with methane hydrate in our calculation. This

573 value is much higher than the methane solubility of the water and the amount produced

574 by the serpentinization of the core. Hence, our hypothesis requires methane ice and/or 
methane hydrate accretion when moons form.

576 The thermal evolution of icy bodies when such multiple gases are dissolved in the ocean

577 is a future work.

\section{5. Conclusion}

579 We investigate the conditions under which a thick subsurface ocean could be achieved

580 in Mimas and Enceladus, and Mimas is found to have a thicker thermal lithosphere than

581 that of Enceladus in a one-dimensional thermal evolution simulation. In our calculation,

582 the current shell thicknesses of Mimas and Enceladus are achieved within almost 100

583 Myr since both moons are small. If a methane hydrate layer exists beneath the ice layer,

584 the final ocean thickness is thicker than that in the case without methane hydrate

585 because the methane hydrate layer plays a role in shell insulation. If there is a methane

586 hydrate layer, $\sim 2 \mathrm{GW}$ is needed to sustain more than $50 \mathrm{~km}$ of subsurface ocean on

587 Mimas, and $\sim 7.5 \mathrm{GW}$ is needed for more than $25 \mathrm{~km}$ ocean on Enceladus. These

588 values are consistent with the tidal heating rate evaluated by the orbital conditions of

589 Mimas and Enceladus. Additionally, the thermal lithosphere of Mimas is thicker than

590 that of Enceladus if the tidal heating rate for Mimas is less than $\sim 20 \mathrm{GW}$. The surface 
591 heat flux is depressed if there is a methane hydrate layer, which is consistent with the

592 unrelaxed surface of Mimas. It is assumed that a methane hydrate layer explains the

593 thick subsurface oceans of both moons and the inactive shell of Mimas. Although we

594 need a more detailed investigation of the tidal heating rate since it depends on an orbital

595 evolution that we do not consider in this study, methane hydrate may be a key

596 compound for understanding the histories of icy moons.

597

598 Declarations

599

Ethics approval and consent to participate

600

Not applicable

601

Consent for publication

602

Not applicable

603

List of abbreviations

604

CIRS: Composite infrared spectrometer; MMR: Mean motion resonance.

605

Availability of data and materials

606

The datasets used and/or analyzed during the current study are available 
No conflict of interest

\section{Funding}

This study was supported by KAKENHI from the Japan Society for the

Promotion of Science (Grant No. JP17K05635 and JP17H06456). work. All authors read and approved the manuscript. 


\section{References}

625 Běhounková M, Tobie G, Choblet G, Čadek, O (2013) Impact of tidal heating on the

626 onset of convection in Enceladus's ice shell. Icarus 226(1):898-904.

627 Bland MT, Singer KN, McKinnon WB, Schenk PM (2012) Enceladus' extreme heat

628 flux as revealed by its relaxed craters. Geophysical Research Letters 39(17)

629 Bouquet A, Mousis O, Waite JH, Picaud S (2015) Possible evidence for a methane

630 source in Enceladus' ocean. Geophysical Research Letters 42(5):1334-1339

631 Čadek O, Tobie G, Van Hoolst T, Massé M, Choblet G, Lefèvre A, Mitri G, Baland

632 RM, Běhounková M, Bourgeois O, Trinh A (2016) Enceladus's internal ocean and ice

633 shell constrained from Cassini gravity, shape, and libration data. Geophysical Research

634 Letters 43(11):5653-5660

635 Chen E, Nimmo F, Glatzmaier G (2014) Tidal heating in icy satellite oceans. Icarus

$636 \quad 229: 11-30$

637 Charnoz S, Crida A, Castillo-Roge, J C, Lainey V, Dones L, Karatekin O, Tobie G,

638 Mathis S, Le Poncin-Lafitte C, \& Salmon J. (2011) Accretion of Saturn's mid-sized 
639 moons during the viscous spreading of young massive rings: Solving the paradox of

640 silicate-poor rings versus silicate-rich moons. Icarus 216:535-550.

641 Choblet G, Tobie G, Sotin C, Běhounková M, Čadek O, Postberg F, Souček

642 O (2017) Powering prolonged hydrothermal activity inside Enceladus.

643 Nature Astronomy 1(12):841-847

644 Choukroun M, Grasset O (2010) Thermodynamic data and modeling of the water and

645 ammonia-water phase diagrams up to $2.2 \mathrm{GPa}$ for planetary geophysics. The Journal of

646 Chemical Physics 133(14):144502

647 Choukroun M, Grasset O, Tobie G, Sotin C (2010) Stability of methane clathrate

648 hydrates under pressure: Influence on outgassing processes of methane on Titan. Icarus

$649 \quad 205(2): 581-593$

650 Duan Z, Mao S (2006) A thermodynamic model for calculating methane solubility,

651 density and gas phase composition of methane-bearing aqueous fluids from 273 to

652 523K and from 1 to 2000bar. Geochimica et Cosmochimica Acta 70(13):3369-3386

653 Durham WB, Kirby SH, Stern LA, Zhang W (2003) The strength and rheology of

654 methane clathrate hydrate. Journal of Geophysical Research: Solid Earth 108(B4) 
655 Fuller J, Luan J, Quataert E (2016) Resonance locking as the source of rapid tidal

656 migration in the Jupiter and Saturn moon systems. Monthly Notices of the Royal

657 Astronomical Society 458(4):3867-3879

658 Goldsby DL, Kohlstedt DL (2001) Superplastic deformation of ice: Experimental

659 observations. Journal of Geophysical Research: Solid Earth 106(B6):11017-11030

660 Hand KP, Khurana KK, Chyba CF (2011) Joule heating of the south polar terrain on

661 Enceladus. Journal of Geophysical Research 116(E4)

662 Hobbs PV (2010) Ice physics. Oxford university press

663 Howett CJA, Spencer JR, Pearl J, Segura M (2011) High heat flow from Enceladus'

664 south polar region measured using $10-600 \mathrm{~cm}^{-1}$ Cassini/CIRS data. Journal of

665 Geophysical Research 116(E3)

666 Hsu HW, Postberg F, Sekine Y, Shibuya T, Kempf S, Horányi M, Juhász A, Altobelli

667 N, Suzuki K, Masaki Y, Kuwatani T, Tachibana S, Sirono Si, Moragas-Klostermeyer G,

668 Srama R (2015) Ongoing hydrothermal activities within Enceladus. Nature

$669 \quad 519(7542): 207-210$

670 Iess L, Stevenson DJ, Parisi M, Hemingway D, Jacobson RA, Lunine JI, Nimmo F, 
671 Armstrong JW, Asmar SW, Ducci M, Tortora P (2014) The Gravity Field and Interior

672 Structure of Enceladus. Science 344(6179):78-80

673 Kalousová K, Sotin C (2020) The Insulating Effect of Methane Clathrate Crust on

674 Titan's Thermal Evolution. Geophysical Research Letters 47(13): e2020GL087481.

675 Kamata S (2018) One-Dimensional Convective Thermal Evolution Calculation Using a

676 Modified Mixing Length Theory: Application to Saturnian Icy Satellites. Journal of

677 Geophysical Research: Planets 123(1):93-112

678 Kamata S, Nimmo F, Sekine Y, Kuramoto K, Noguchi N, Kimura J, Tani A (2019)

679 Pluto's ocean is capped and insulated by gas hydrates. Nature Geoscience 12(6):407-

$680 \quad 410$

681 Kimura J, Kamata S (2020) Stability of the subsurface ocean of pluto. Planetary and

682 Space Science 181:104828

683 Kirchoff MR, Schenk P (2009) Crater modification and geologic activity in Enceladus'

684 heavily cratered plains: Evidence from the impact crater distribution. Icarus

$685 \quad 202(2): 656-668$

686 Lainey V, Karatekin Ö, Desmars J, Charnoz S, Arlot JE, Emelyanov N, Le Poncin- 
687 Lafitte C, Mathis S, Remus F, Tobie G, Zahn JP (2012) Strong tidal dissipation in

688 Saturn and constraints on Enceladus' thermal state. The Astrophysical Journal

$689752(1): 14$

690 Leliwa- Kopystyński J, Maruyama M, Nakajima T (2002) The water-ammonia phase

691 diagram up to $300 \mathrm{MPa}$ : Application to icy satellites. Icarus 159(2):518-528

692 Lodders K (2003) Solar system abundances and condensation temperatures of the

693 elements. The Astrophysical Journal 591(2):1220

694 Malamud U, Prialnik D (2013) Modeling serpentinization: Applied to the early

695 evolution of Enceladus and Mimas. Icarus 225(1):763-774

696 McCollom TM (2016) Abiotic methane formation during experimental serpentinization

697 of olivine. Proceedings of the National Academy of Sciences 113(49):13965-13970

698 McCollom TM, Seewald JS (2007) Abiotic Synthesis of Organic Compounds in Deep-

699 Sea Hydrothermal Environments. Chemical Reviews 107(2):382-401,

700 Meyer J, Wisdom J (2007) Tidal heating in Enceladus. Icarus 188(2):535-539,

701 Meyer J, Wisdom J (2008) Tidal evolution of Mimas, Enceladus, and Dione. Icarus

702 193(1): 213-22. 
703 Mumma MJ, Charnley SB (2011) The Chemical Composition of Comets-Emerging

704 Taxonomies and Natal Heritage. Annual Review of Astronomy and Astrophysics

705 49(1):471-524

706 Neveu M, Rhoden A (2017) The origin and evolution of a differentiated Mimas. Icarus

$707 \quad 296: 183-196$

708 Nimmo F, Spencer JR, Pappalardo RT, Mullen ME (2007) Shear heating as the origin

709 of the plumes and heat flux on Enceladus. Nature 447(7142):289-291

710 Nimmo F, Barr AC, Běhounková M, McKinnon WB (2018) The thermal and orbital

711 evolution of Enceladus: observational constraints and models. Enceladus and the Icy

712 Moons of Saturn 475:79-94

713 Noyelles B (2017) Interpreting the librations of a synchronous satellite - How their

714 phase assesses Mimas’ global ocean. Icarus 282:276-289

715 Porco CC, Helfenstein P, Thomas P, Ingersoll A, Wisdom J, West R, Neukum G, Denk

716 T, Wagner R, Roatsch T, et al (2006) Cassini Observes the Active South Pole of

717 Enceladus. Science 311(5766):1393-1401

718 Rhoden AR, Henning W, Hurford TA, Patthoff DA, Tajeddine R (2017) The 
719 implications of tides on the Mimas ocean hypothesis. Journal of Geophysical Research:

720 Planets 122(2):400-410

721 Roberts JH, Nimmo F (2008) Tidal heating and the long-term stability of a subsurface

722 ocean on Enceladus. Icarus 194(2):675-689

723 Schubert G, Anderson JD, Travis BJ, Palguta J (2007) Enceladus: Present internal

724 structure and differentiation by early and long-term radiogenic heating. Icarus

$725 \quad 188(2): 345-355$

726 Segatz M, Spohn T, Ross M, Schubert G (1988) Tidal dissipation, surface heat flow,

727 and figure of viscoelastic models of Io. Icarus 75(2):187-206

728 Sloan Jr ED, Koh CA (2007) Clathrate hydrates of natural gases. CRC press

729 Souček O, Běhounková M, Čadek O, Hron J, Tobie G, Choblet G. (2019). Tidal

730 dissipation in Enceladus' uneven, fractured ice shell. Icarus 328:218-231.

731 Tajeddine R, Rambaux N, Lainey V, Charnoz S, Richard A, Rivoldini A, Noyelles B

732 (2014) Constraints on Mimas' interior from Cassini ISS libration measurements.

733 Science 346(6207):322-324

734 Taubner RS, Pappenreiter P, Zwicker J, Smrzka D, Pruckner C, Kolar P, Bernacchi S, 
735 Seifert AH, Krajete A, Bach W, Peckmann J, Paulik C, Firneis MG, Schleper C,

736 Rittmann SKMR (2018) Biological methane production under putative Enceladus-like

737 conditions. Nature Communications 9(1):748

738 Thomas P, Tajeddine R, Tiscareno M, Burns J, Joseph J, Loredo T, Helfenstein P,

739 Porco C (2016) Enceladus's measured physical libration requires a global subsurface

740 ocean. Icarus 264:37-47

741 Tian Z, Nimmo F (2019) Implications of Second Order Resonance for the Thermal and

742 Orbital Evolution of Mimas. Monthly Notices of the Royal Astronomical Society

743 492(1):369-376.

744 Tobie G, Choblet G, Sotin C (2003) Tidally heated convection: Constraints on Europa's

745 ice shell thickness. Journal of Geophysical Research 108(E11):5124

746 Vu TH, Gloesener E, Choukroun M, Ibourichene A, Hodyss R (2014) Experimental

747 Study on the Effect of Ammonia on the Phase Behavior of Tetrahydrofuran Clathrates.

748 The Journal of Physical Chemistry B 118(47):13371-13377

749 Waite JH, Glein CR, Perryman RS, Teolis BD, Magee BA, Miller G, Grimes J, Perry

750 ME, Miller KE, Bouquet A, Lunine JI, Brockwell T, Bolton SJ (2017) Cassini finds 
751 molecular hydrogen in the Enceladus plume: Evidence for hydrothermal processes.

752 Science 356(6334):155-159

753 Waite WF, Stern LA, Kirby SH, Winters WJ, Mason DH (2007) Simultaneous

754 determination of thermal conductivity, thermal diffusivity and specific heat in sI

755 methane hydrate. Geophysical Journal International 169(2):767-774

756 Waite WF, Santamarina JC, Cortes DD, Dugan B, Espinoza DN, Germaine J, Jang J,

757 Jung JW, Kneafsey TJ, Shin H, Soga K, Winters WJ, Yun TS (2009) Physical

758 properties of hydrate-bearing sediments. Reviews of Geophysics 47(4)

759 


\section{Figures}

761 Figure 1; Interior structure models of Enceladus and Mimas; These are snapshots of the

762 interior structure of Enceladus and Mimas in a case where methane hydrate exists. From

763 top to bottom, each layer represents ice, methane hydrate, ocean, and a rocky core.

764 Figure 2; Shell thickness evolution of Mimas with time; The changes in shell thickness

765 of Mimas under $3 \mathrm{GW}$ for tidal heating, $573 \mathrm{~K}$ for initial core temperature, and different

766 initial methane concentrations are shown. (a) is the case of $1000 \mathrm{~mol} \mathrm{~m}^{-3}$ for initial

767 methane concentration and (b) is the case of $0 \mathrm{~mol} \mathrm{~m}^{-3}$ (in other words, only ice exists).

768 Each line indicates the boundary of each layer. The colour contour in both figures

769 represents the ratio of $F_{\text {conv }}$ to the total heat flux $\left(=F_{\text {conv }}+F_{\text {cond }}\right)$. The coloured region

770 shows the area in which the convection occurs.

771 Figure 3; The differences in shell thickness evolution among different initial methane

772 concentrations for Mimas; The final structures of Mimas under different initial methane

773 concentrations are shown. The initial core temperature is $573 \mathrm{~K}$, and the tidal heating rate

774 is $3 \mathrm{GW}$.

775 Figure 4; The dependency of final shell thickness and thermal lithosphere on tidal heating 
776 rate; The final thickness of the shell and ocean and thermal lithosphere under different

777 tidal heating rates are shown. The initial core temperature for Mimas is $573 \mathrm{~K}$ and that

778 for Enceladus is 1273 K. (a) is the case with the methane hydrate layer for Mimas, that

779 is, $1000 \mathrm{~mol} \mathrm{~m}^{-3}$ initial methane concentration, (b) is the case without the methane

780 hydrate layer for Mimas, that is, $0 \mathrm{~mol} \mathrm{~m}^{-3}$ initial methane concentration, (c) is the case

781 with the methane hydrate layer for Enceladus, and (d) is the case without the methane

782 hydrate layer for Enceladus. The vertical axis represents the sum of the shell and ocean.

783 The horizontal axis represents the added tidal heating rate. In the area painted red in the

784 figure, convection occurs; in other words, $F_{\text {conv }}$ is not 0 . The thermal lithosphere means

785 that convection does not occur in the region, which is the white region of the shell in the

786 figure.

787 Figure 5; Surface heat flux on Mimas; The current surface heat flux of Mimas is shown

788 under an initial core temperature of $573 \mathrm{~K}$. The solid line represents the case with methane

789 hydrate, that is, the initial methane concentration is $1000 \mathrm{~mol} \mathrm{~m}^{-3}$. The dashed line

790 represents the case without methane hydrate, that is, the initial methane concentration is

$7911000 \mathrm{~mol} \mathrm{~m}^{-3}$. 
792 Figure 6; Summary of the results; The final ocean thicknesses for Mimas and Enceladus

793 under different tidal heating rates are summarized. (a) is the case without the methane

794 hydrate layer, that is, the initial methane concentration is $0 \mathrm{~mol} \mathrm{~m}^{-3}$, and (b) is the case

795 with the methane hydrate layer, that is, the initial methane concentration is 1000

$796 \mathrm{~mol} \mathrm{~m}^{-3}$. The vertical axis shows the tidal heating rate for Enceladus, and the horizontal

797 axis shows that for Mimas. Each tidal heating rate can correspond to the final ocean

798 thickness which is described at the opposite axis. The dashed lines correspond to $50 \mathrm{~km}$

799 of the final ocean thickness for Mimas and to $25 \mathrm{~km}$ for Enceladus. The area surrounded

800 by a dashed line means that a thick ocean exists in each moon. The grey area represents

801 that at least one satellite - Mimas or Enceladus - does not have an ocean. The red area

802 represents that the final lithosphere of Mimas is thicker than that of Enceladus. The blue

803 area represents that the lithosphere of Mimas is thinner than that of Enceladus, although

804 both moons have the inner ocean.

805

806 Preparing Tables

807 Table 1 The thermal and physical properties of Mimas and Enceladus. 


\begin{tabular}{lllll}
\hline Parameter & Symbol & Units & Mimas & Enceladus \\
\hline Mean surface radius & $R$ & $\mathrm{~km}$ & 198.2 & 252.0 \\
Bulk density & $\rho$ & $\mathrm{kg} \mathrm{m}^{-3}$ & 1149 & 1611 \\
Surface temperature & $T_{\text {surf }}$ & $\mathrm{K}$ & 76 & 59 \\
Semimajor axis & $a$ & $\mathrm{~km}$ & 185.539 & 237.948 \\
Orbital eccentricity & $e$ & & 0.0196 & 0.0047 \\
\hline
\end{tabular}

808 Table 2 Parameters used in numerical simulation.

\begin{tabular}{|c|c|c|c|}
\hline & Symbo & & \\
\hline Parameter & 1 & Units & Value \\
\hline Density of ice & $\rho_{\text {ice }}$ & $\mathrm{kg} \mathrm{m}^{-3}$ & 920 \\
\hline Density of methane hydrate & $\rho_{\text {hyd }}$ & $\mathrm{kg} \mathrm{m}^{-3}$ & 920 \\
\hline Density of ocean & $\rho_{\text {oce }}$ & $\mathrm{kg} \mathrm{m}^{-3}$ & 1000 \\
\hline Density of rocky core & $\rho_{\text {core }}$ & $\mathrm{kg} \mathrm{m}^{-3}$ & 2450 \\
\hline Core radius of Mimas & $r_{\mathrm{c}, \mathrm{mim}}$ & $\mathrm{km}$ & 98.9 \\
\hline Core radius of Enceladus & $r_{\mathrm{c}, \mathrm{enc}}$ & $\mathrm{km}$ & 190.7 \\
\hline Specific heat of rocky core & $C_{\mathrm{p}, \text { core }}$ & $\mathrm{J} \mathrm{kg}^{-1} \mathrm{~K}^{-1}$ & 1000 \\
\hline Specific heat of ice & $C_{\mathrm{p}, \text { ice }}$ & $\mathrm{J} \mathrm{kg}^{-1} \mathrm{~K}^{-1}$ & 2100 \\
\hline Specific heat of methane hydrate & $C_{\mathrm{p}, \mathrm{hyd}}$ & $\mathrm{J} \mathrm{kg}^{-1} \mathrm{~K}^{-1}$ & 2100 \\
\hline Thermal conductivity of rocky core & $k_{\text {core }}$ & $\mathrm{W} \mathrm{m} \mathrm{m}^{-1} \mathrm{~K}^{-1}$ & 3.0 \\
\hline Thermal conductivity of ice & $k_{\text {ice }}$ & $\mathrm{W} \mathrm{m} \mathrm{m}^{-1} \mathrm{~K}^{-1}$ & 2.6 \\
\hline $\begin{array}{l}\text { Thermal conductivity of methane } \\
\text { hydrate }\end{array}$ & $k_{\text {hyd }}$ & $\mathrm{W} \mathrm{m} \mathrm{m}^{-1} \mathrm{~K}^{-1}$ & 0.6 \\
\hline Activation energy of ice & $E_{\mathrm{a}, \mathrm{ice}}$ & $\mathrm{J} \mathrm{mol}^{-1}$ & 60000 \\
\hline Activation energy of methane hydrate & $E_{\text {a,hyd }}$ & $\mathrm{J} \mathrm{mol}^{-1}$ & 90000 \\
\hline Reference viscosity of ice & $\eta_{\text {refice }}$ & Pa s & $1.0 \times 10^{14}$ \\
\hline Reference viscosity of methane hydrate & $\eta_{\text {ref,hyd }}$ & Pa s & $2.0 \times 10^{15}$ \\
\hline Thermal expansivity of ice & $\alpha_{\text {ice }}$ & $\mathrm{K}^{-1}$ & $10^{-4}$ \\
\hline
\end{tabular}


Thermal expansivity of methane hydrate $\begin{array}{cccc}\alpha_{\text {hyd }} & \mathrm{K}^{-1} & 10^{-4}\end{array}$

Latent heat of ice $\quad L_{\text {ice }} \quad \mathrm{J} \mathrm{kg}^{-1} \quad 333000$

Latent heat of methane hydrate $\quad L_{\mathrm{hyd}} \quad \mathrm{J} \mathrm{kg}^{-1} \quad 437000$

809 Table 3 The concentration and properties of radioactive elements.

\begin{tabular}{llll}
\hline & Concentration $(\mathrm{ppb})$ & Decay energy $(\mathrm{W} / \mathrm{kg})$ & Half life time $(\mathrm{Myr})$ \\
\hline${ }^{238 \mathrm{U}}$ & 19.9 & $94.65 \times 10^{-6}$ & 4468 \\
${ }^{235 \mathrm{U}}$ & 5.4 & $568.7 \times 10^{-6}$ & 703.81 \\
\multirow{2}{*}{${ }^{232} \mathrm{Th}$} & 38.7 & $26.38 \times 10^{-6}$ & 14030 \\
${ }^{40} \mathrm{~K}$ & 738 & $29.17 \times 10^{-6}$ & 1277 \\
\hline
\end{tabular}

810 
Figures

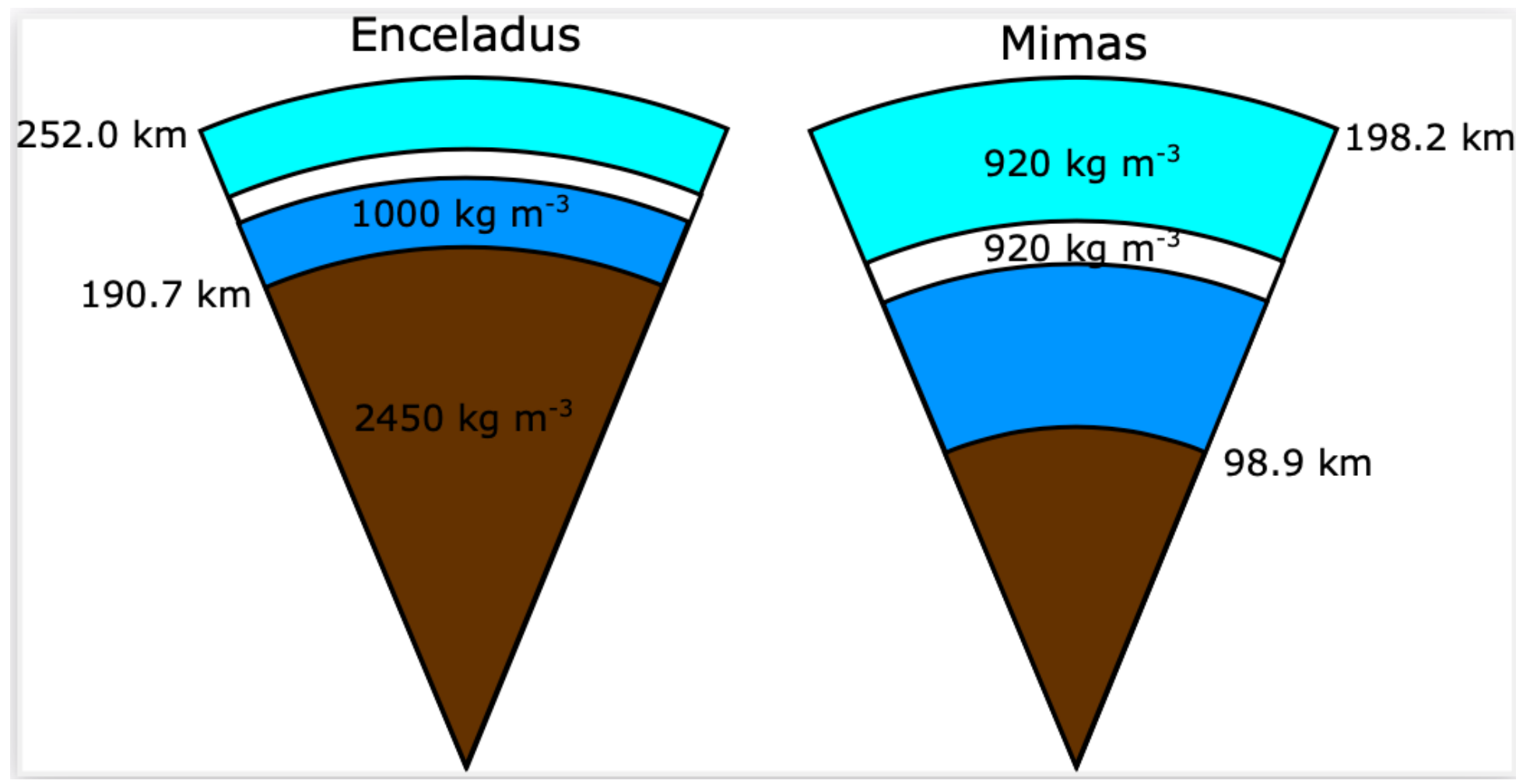

Figure 1

Interior structure models of Enceladus and Mimas; These are snapshots of the interior structure of Enceladus and Mimas in a case where methane hydrate exists. From top to bottom, each layer represents ice, methane hydrate, ocean, and a rocky core.

(a)

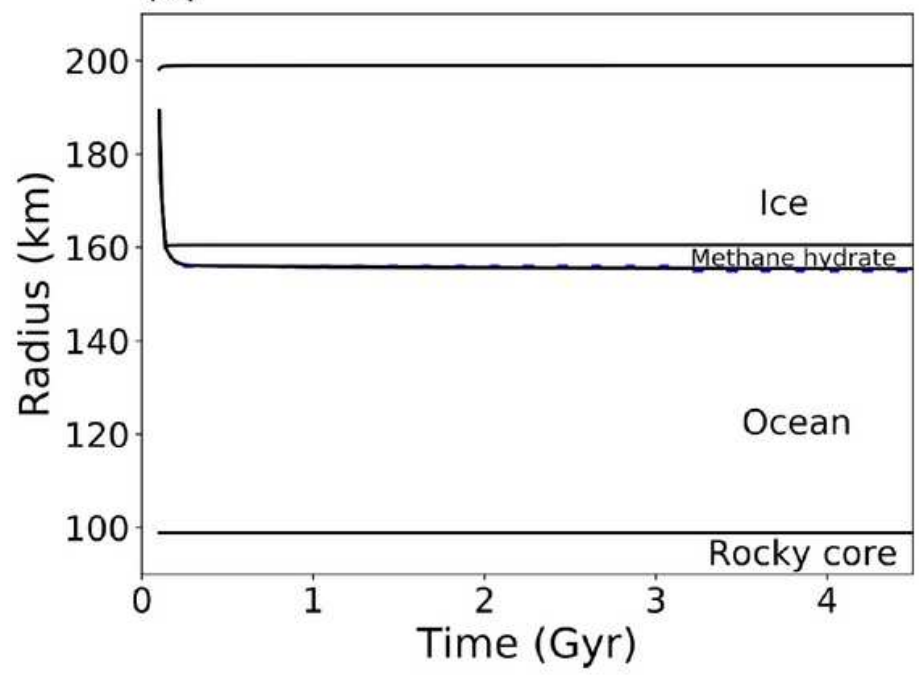

(b)

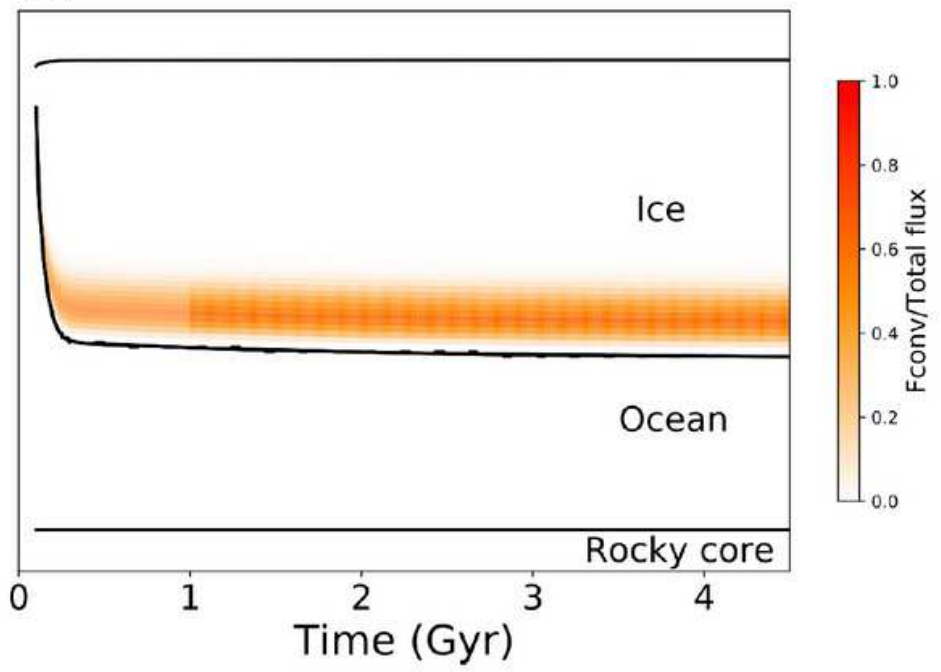

Figure 2 
Shell thickness evolution of Mimas with time; The changes in shell thickness of Mimas under $3 \mathrm{GW}$ for tidal heating, $573 \mathrm{~K}$ for initial core temperature, and different initial methane concentrations are shown. (a) is the case of $1000 \mathrm{~mol} \mathrm{~m}^{\wedge}(-3)$ for initial methane concentration and (b) is the case of $0 \mathrm{~mol} \mathrm{~m}^{\wedge}(-3)$ (in other words, only ice exists). Each line indicates the boundary of each layer. The colour contour in both figures represents the ratio of $F_{-}$(conv ) to the total heat flux (=F_(conv )+F_(cond )). The coloured region shows the area in which the convection occurs.

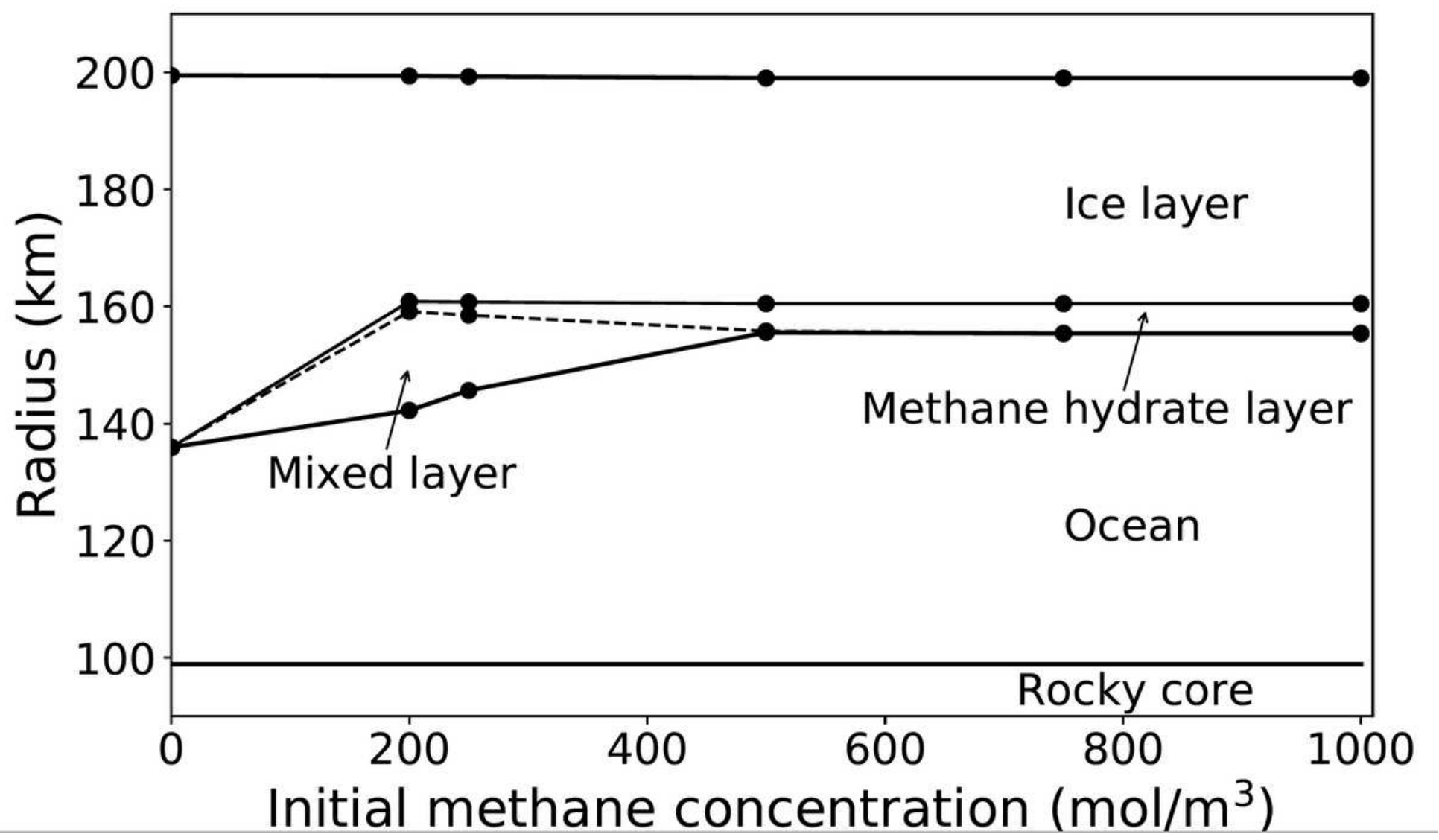

Figure 3

The differences in shell thickness evolution among different initial methane concentrations for Mimas; The final structures of Mimas under different initial methane concentrations are shown. The initial core temperature is $573 \mathrm{~K}$, and the tidal heating rate is $3 \mathrm{GW}$. 

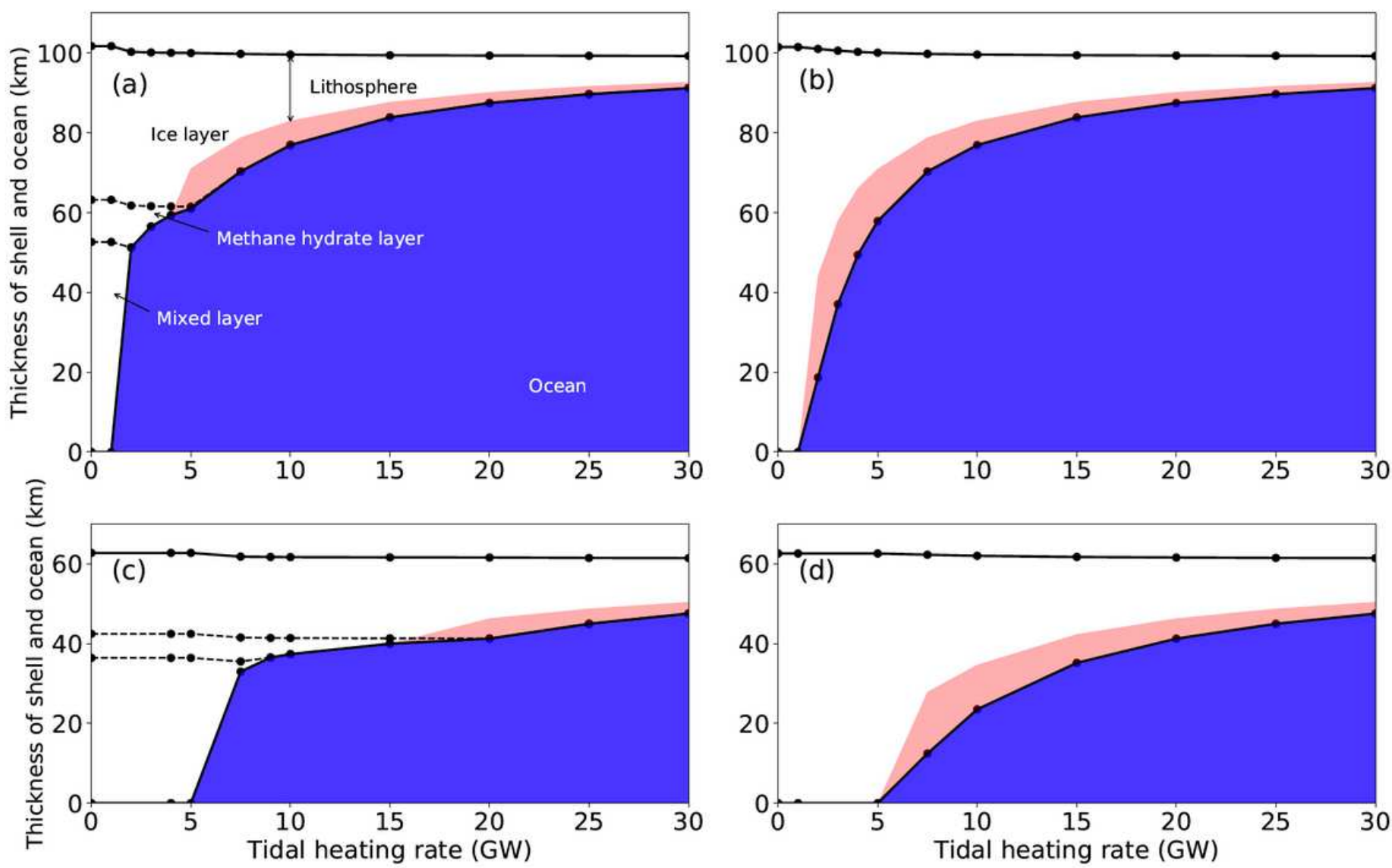

\section{Figure 4}

The dependency of final shell thickness and thermal lithosphere on tidal heating rate; The final thickness of the shell and ocean and thermal lithosphere under different tidal heating rates are shown. The initial core temperature for Mimas is $573 \mathrm{~K}$ and that for Enceladus is $1273 \mathrm{~K}$. (a) is the case with the methane hydrate layer for Mimas, that is, $1000 \mathrm{~mol} \mathrm{~m} \wedge^{\wedge}(-3)$ initial methane concentration, (b) is the case without the

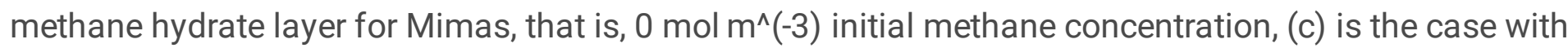
the methane hydrate layer for Enceladus, and (d) is the case without the methane hydrate layer for Enceladus. The vertical axis represents the sum of the shell and ocean. The horizontal axis represents the added tidal heating rate. In the area painted red in the figure, convection occurs; in other words, $F_{-}$(conv ) is not 0 . The thermal lithosphere means that convection does not occur in the region, which is the white region of the shell in the figure. 


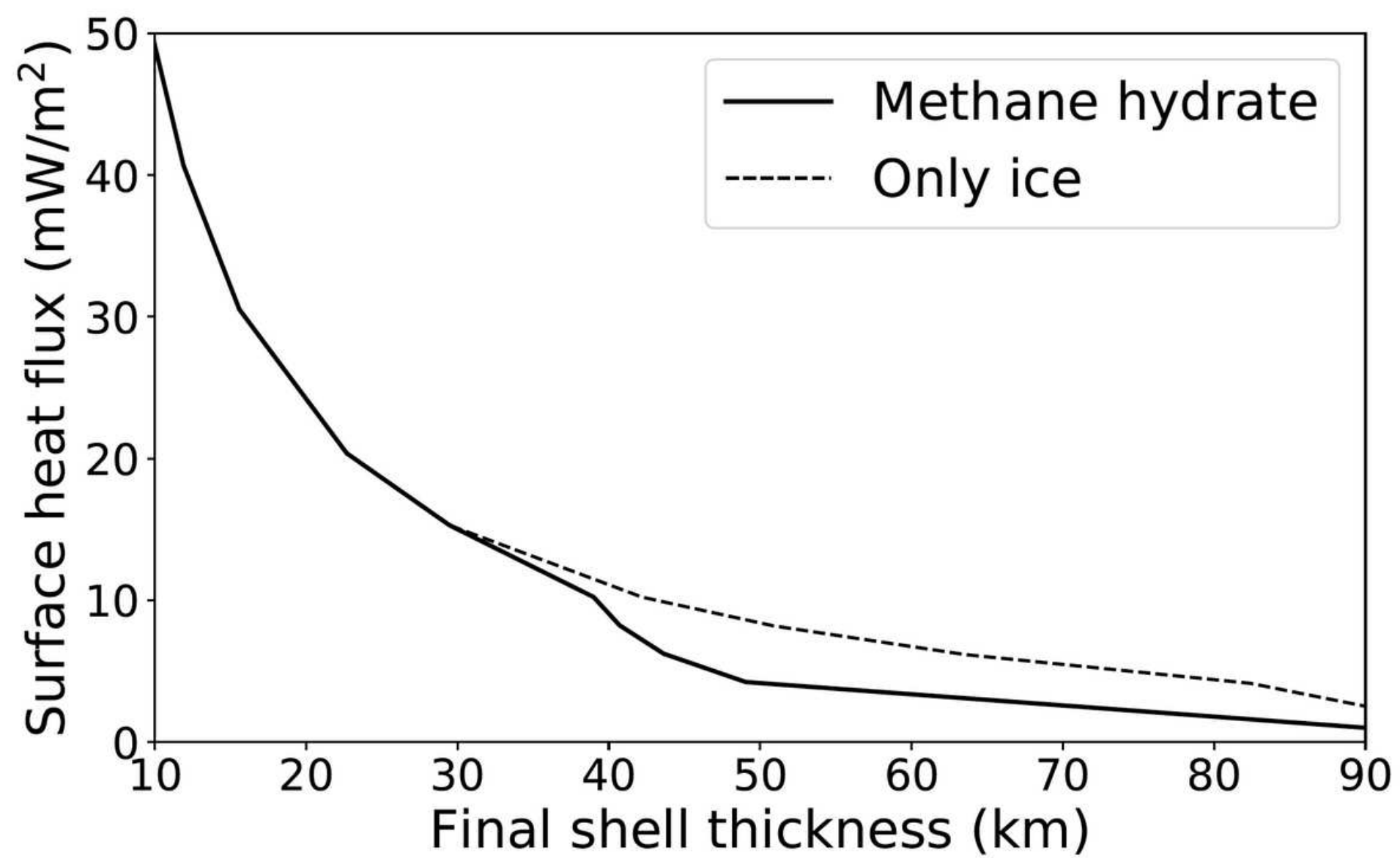

Figure 5

Surface heat flux on Mimas; The current surface heat flux of Mimas is shown under an initial core temperature of $573 \mathrm{~K}$. The solid line represents the case with methane hydrate, that is, the initial methane concentration is $1000 \mathrm{~mol} \mathrm{~m}^{\wedge}(-3)$. The dashed line represents the case without methane hydrate, that is, the initial methane concentration is $1000 \mathrm{~mol} \mathrm{~m}^{\wedge}(-3)$.

(a) Final ocean thickness of Mimas $(\mathrm{km})$

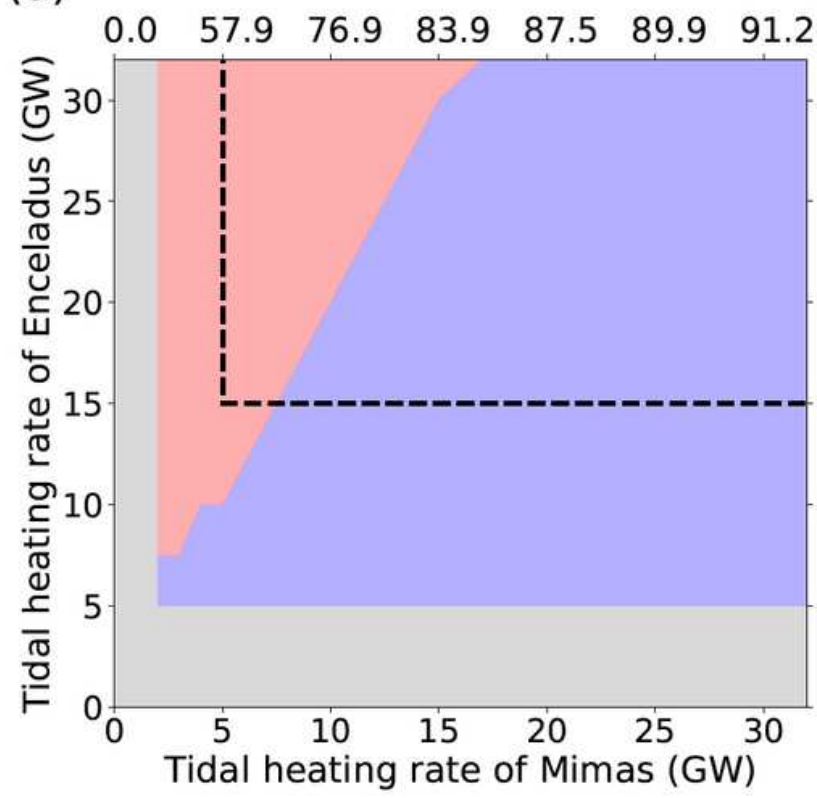

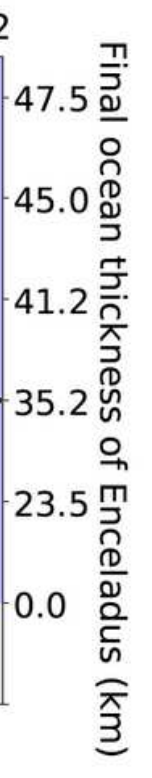

(b)

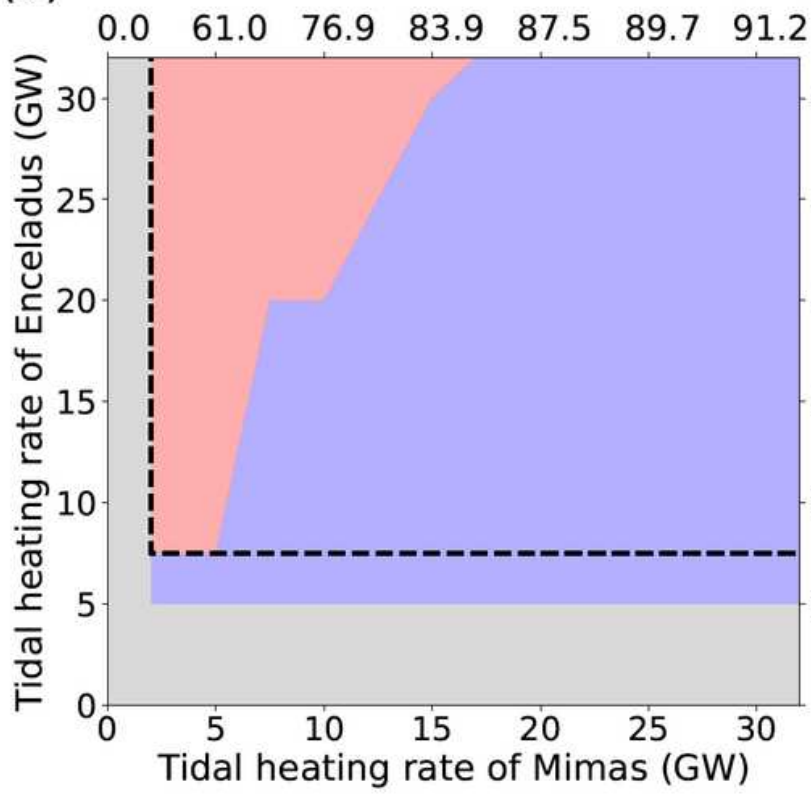

Final ocean thickness of Mimas $(\mathrm{km})$

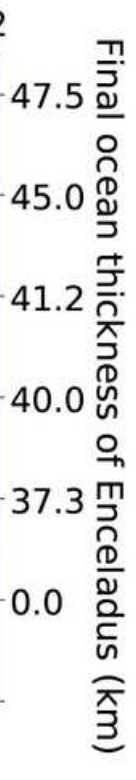




\section{Figure 6}

Summary of the results; The final ocean thicknesses for Mimas and Enceladus under different tidal heating rates are summarized. (a) is the case without the methane hydrate layer, that is, the initial methane concentration is $0 \mathrm{~mol} \mathrm{~m}^{\wedge}(-3)$, and (b) is the case with the methane hydrate layer, that is, the initial methane concentration is $1000 \mathrm{~mol} \mathrm{~m}^{\wedge}(-3)$. The vertical axis shows the tidal heating rate for Enceladus, and the horizontal axis shows that for Mimas. Each tidal heating rate can correspond to the final ocean thickness which is described at the opposite axis. The dashed lines correspond to $50 \mathrm{~km}$ of the final ocean thickness for Mimas and to $25 \mathrm{~km}$ for Enceladus. The area surrounded by a dashed line means that a thick ocean exists in each moon. The grey area represents that at least one satellite Mimas or Enceladus - does not have an ocean. The red area represents that the final lithosphere of Mimas is thicker than that of Enceladus. The blue area represents that the lithosphere of Mimas is thinner than that of Enceladus, although both moons have the inner ocean.

\section{Supplementary Files}

This is a list of supplementary files associated with this preprint. Click to download.

- GraphycalabstractNishitani.png 